\title{
GEOMETRY OF STRICTLY CONVEX DOMAINS AND AN APPLICATION TO THE UNIFORM ESTIMATE OF THE $\bar{\partial}$-PROBLEM
}

\author{
TEN-GING CHEN
}

\begin{abstract}
In this paper, we construct a nice defining function $\rho$ for a bounded smooth strictly convex domain $\Omega$ in $R^{n}$ with explicit gradient and Hessian estimates near the boundary $\partial \Omega$ of $\Omega$. From the approach, we deduce that any two normals through $\partial \Omega$ do not intersect in any tubular neighborhood of $\partial \Omega$ with radius which is less than $\frac{1}{K}$, where $K$ is the maximum principal curvature of $\partial \Omega$. Finally, we apply such $\rho$ to obtain an explicit upper bound of the constant $C_{\Omega}$ in the Henkin's estimate $\left\|H_{\Omega} f\right\|_{L^{\infty}(\Omega)} \leq C_{\Omega}\|f\|_{L^{\infty}(\Omega)}$ of the $\bar{\partial}$-problem on strictly convex domains $\Omega$ in $\mathbf{C}^{n}$.
\end{abstract}

\section{INTRODUCTION}

Given a bounded smooth domain $\Omega \subset \mathbf{C}^{n}$, one would like to solve the following $\bar{\partial}$-problem:

$$
\begin{aligned}
& \text { Let } f=\sum_{j=1}^{n} f_{j} d \overline{z_{j}} \text { be a smooth }(0,1) \text { - } \\
& \text { form and } \bar{\partial} \text {-closed on } \Omega \text {, i.e., } \bar{\partial} f=0 \text {. }
\end{aligned}
$$

Does $\bar{\partial} u=f$ hold for some $u \in C^{\infty}(\Omega)$ ?

In fact, it is well known that $(0.1)$ can always be solved for any pseudoconvex domain $\Omega$ in $\mathbf{C}^{n}$ without any boundary restrictions on $\Omega$ [7, p. 87]. Therefore, we may restrict ourself to the category of pseudoconvex domains $\Omega$ and ask further:

$(0.2)$ If $f \in L_{(0,1)}^{p}(\Omega)$, i.e., a $(0,1)$-form with coefficients in $L^{p}(\Omega)$, and $\bar{\partial} f=$ 0 , where $1 \leq p \leq \infty$, then can one solve (0.1) with the following inequality:

$$
\|u\|_{L^{p}(\Omega)} \leq C_{\Omega}\|f\|_{L^{p}(\Omega)},
$$

where $C_{\Omega}$ is a constant independent of $f$ ?

For bounded pseudoconvex domains, this is known for $p=2[6, \mathrm{p} .107]$ and $C_{\Omega}=\sqrt{e} \cdot R$, where $R$ is the diameter of $\Omega$ and $e=2.71828 \ldots$ the natural exponential. For $p=\infty$, Sibony [12, p. 239] proved

Received by the editors January 25, 1994 and, in revised form, August 30, 1994.

1991 Mathematics Subject Classification. Primary 32F20, 32A25; Secondary 52A20, 53A07, $53 \mathrm{C} 99$.

Key words and phrases. Strictly convex domain, principal curvature, $\bar{\partial}$-problem.

This paper is a part of the author's doctoral dissertation with some minor modification which was directed by Professor H. Wu at the University of California at Berkeley. 
(0.3) There exists a bounded, smooth pseudoconvex domain $\Omega$ in $\mathbf{C}^{3}$ and a smooth $\bar{\partial}$-closed $(0,1)$-form $f$ on $\Omega$ which is continuous on $\bar{\Omega}$ such that any solution of $\bar{\partial} u=f$ must satisfy $\|u\|_{L^{\infty}(\Omega)}=\infty$.

Therefore, $(0.2)$ fails for $p=\infty$. In general, (0.2) holds in the case of bounded, smooth strongly pseudoconvex domains $\Omega$ in any Stein manifold [8, p. 302]. Of particular interest is the case of $p=\infty$ in $\mathbf{C}^{n}$; the solution in this case was first given by Grauert-Lieb [4] and Henkin [5] independently in 1970. We are mainly interested in Henkin's solution in this paper because it has the virtue of being more explicit.

Henkin's method first constructs an integral representation formula for a solution $H_{\Omega} f$ of $\bar{\partial} u=f$ and proceeds to make estimates of the integral to arrive at the inequality $\left\|H_{\Omega} f\right\|_{L^{\infty}(\Omega)} \leq C_{\Omega}\|f\|_{L^{\infty}(\Omega)}$. More precisely, he proved (0.4) Integral representation for the $\bar{\partial}$-problem [5]. Let $f \in C_{(0,1)}^{\infty}(\bar{\Omega})$ and $\bar{\partial} f=0$ on $\Omega$. Then the function,

$$
\left(H_{\Omega} f\right)(z)=\int_{\partial \Omega \times[0,1]} f(\zeta) \wedge H_{1}(z, \zeta, \lambda)-\int_{\Omega} f(\zeta) \wedge H_{2}(z, \zeta),
$$

where $H_{1}(z, \zeta, \lambda)$ and $H_{2}(z, \zeta)$ are some definite differential forms constructed by Henkin, satisfies

(i) $H_{\Omega} f \in C^{\infty}(\Omega)$ and $\bar{\partial}\left(H_{\Omega} f\right)=f$ on $\Omega$.

(ii) $\left\|H_{\Omega} f\right\|_{L^{\infty}(\Omega)} \leq C_{\Omega}\|f\|_{L^{\infty}(\Omega)}$, where $C_{\Omega}$ is a constant independent of $f$.

Now, a natural question arises: namely, how does the constant $C_{\Omega}$ depend on the domain? Since Henkin's construction involves many noneffective steps, it seems difficult to make explicit this dependence of $C_{\Omega}$ on $\Omega$. However, in the case of a strictly convex domain $\Omega$ in $\mathbf{C}^{n}$, by studying the geometry of strictly convex domains, we can find an upper bound for $C_{\Omega}$ which turns out to be dependent only on $n, \operatorname{dia}(\Omega), \operatorname{vol}(\Omega)$ and the maximum and minimum principal curvatures of $\partial \Omega$.

There are two sections in this paper. In $\S 1$, we define convexity of $\partial \Omega$ in terms of the second fundamental form of $\partial \Omega$ and study the geometry of strictly convex domains. If $v$ is the unit outward normal of $\partial \Omega, K$ the maximum principal curvature of $\partial \Omega$ and $0 \leq \mu<\frac{1}{K}$, then we apply a result of J. Rauch (1.1) and the Rauch comparison theorem (1.6) to prove the following result (1.2):

(0.5) The restriction of the exponential map of the normal bundle over $\partial \Omega$ to $\widetilde{T}(\mu)$ is a diffeomorphism onto $T(\mu)$, where $\widetilde{T}(\mu)=\{(p,-t v(p)) \mid p \in \partial \Omega, t \in$ $[0, \mu]\}$ and $T(\mu)=\{x \in \bar{\Omega} \mid \operatorname{dist}(x, \partial \Omega) \leq \mu\}$.

Once we have $(0.5)$, we can deduce the smoothness of the signed distance function $\delta$ in $T(\mu)$ easily (1.9). By modifying $\delta$ further, we obtain a natural defining function $\rho$ associated to $\Omega$ with explicit gradient and Hessian estimates in $T(\mu)$ (1.13).

In $\S 2$, we write down the Henkin integral formula $H_{\Omega} f$ for the solution of $\bar{\partial} u=f$ on a smooth bounded strictly convex domain $\Omega$ in $\mathbf{C}^{n}$ and give an explicit uniform estimate $\left\|H_{\Omega} f\right\|_{L^{\infty}(\Omega)} \leq C_{\Omega}\|f\|_{L^{\infty}(\Omega)}$ of the constant $C_{\Omega}$. This can be done by using the defining function $\rho$ constructed in $\S 1$ and Henkin's original ideas. A by-product of the explicit estimate (2.2) is that the stability 
of $C_{\Omega}$ in the case of strictly convex domain can now be proved without handwaving. In fact, by reviewing the whole construction in this paper, we need only $\rho$ to be $C^{3}$, i.e., $\Omega$ is $C^{3}$. Therefore, $C_{\Omega}$ is stable under $C^{3}$ perturbations.

\section{GeOMETRY OF STRICTLY CONVEX DOMAINS IN $\mathbf{R}^{n+1}$}

Let $M$ be a smooth compact hypersurface in $\mathbf{R}^{n+1}$ and $\nabla$ be the Levi-Civita connection associated to the induced metric from the flat metric of $\mathbf{R}^{n+1}$. Then, for any two tangent vector fields $X, Y$ on $M$, we may write

$$
D_{X} Y=\nabla_{X} Y+\mathrm{II}(X, Y) v,
$$

where $D$ is the flat connection on $\mathbf{R}^{n+1}, v$ is the unit outward normal vector field on $M$ and II is the component of $D_{X} Y$ along $v$. The basic property of II is that II is symmetric and $C^{\infty}(M)$-linear in each variable and hence defines a tensor field on $M$. In particular, we can define, for each $p \in M$, a bilinear map

by

$$
\mathrm{II}_{p}: T_{p} M \times T_{p} M \rightarrow \mathbf{R}
$$

$$
\mathrm{II}_{p}\left(X_{p}, Y_{p}\right)=\mathrm{II}(X, Y),
$$

where $X, Y$ are arbitrary extensions of $X_{p}$ and $Y_{p}$, respectively. Thus $\mathrm{II}_{p}$ gives rise to a symmetric bilinear form on $T_{p} M$ for each $p \in M$.

Definition. (1) II is called the second fundamental form of $M$. (2) The eigenvalues of $\mathrm{II}_{p}$ are called the principal curvatures at $p$ and their corresponding eigenvectors are the principal directions.

Now, let $\Omega$ be a bounded smooth domain in $\mathbf{R}^{n+1}$ in the sense that its boundary $\partial \Omega$ is a smooth compact hypersurface in $\mathbf{R}^{n+1}$. Let the second fundamental form of $\partial \Omega$ be II as above. This leads to:

Definition. $\Omega$ is said to be (geometrically) convex if $\mathrm{II}_{p}$ is positive semidefinite for each $p \in \partial \Omega$. If, in addition, $\mathrm{II}_{p}$ is positive definite for all $p \in \partial \Omega$, then we say that $\Omega$ is strictly convex.

Remark. The theorem $(*)$ of Sacksteder $[11$, p. 610] says that the above definition is equivalent to the usual one of convexity.

Definition. A smooth real-valued function $\varphi$ on $\mathbf{R}^{n+1}$ is called a defining function of $\Omega$ if it satisfies the following conditions:

(1) $\Omega=\left\{x \in \mathbf{R}^{n+1} \mid \varphi(x)<0\right\}$,

(2) $\partial \Omega=\left\{x \in \mathbf{R}^{n+1} \mid \varphi(x)=0\right\}$,

(3) $d \varphi(x) \neq 0$ for all $x \in \partial \Omega$.

Remark. Any bounded smooth domain admits at least one defining function which can be constructed by a partition of unity.

From now on, we always assume that $\Omega$ is a bounded smooth strictly convex domain in $\mathbf{R}^{n+1}$ and denote $\partial \Omega$ by $M$. We use $K_{i}(p), i=1,2, \ldots, n$, to denote the principal curvatures at $p$.

Let

$$
K=\max \left\{K_{i}(p) \mid i=1, \ldots, n, p \in M\right\}
$$

and

$$
k=\min \left\{K_{i}(p) \mid i=1, \ldots, n, p \in M\right\}
$$


Then $K$ and $k$ are two positive finite numbers because $M$ is compact.

The main goal of this section is to construct a nice defining function of the domain $\Omega$ which will play an important role in the next section.

First of all, by examining the unit ball in $\mathbf{R}^{n+1}$, we discover that any two normal vectors through its boundary always focus on the origin. In other words, no two normal vectors intersect in any tubular neighborhood of radius which is less than 1. Since $K=1$ for the unit $n$-sphere, this leads to the following general question: For an $\Omega$ and any positive number $\mu<\frac{1}{K}$, is it still true that no two normals through $\partial \Omega$ intersect within the tubular neighborhood of $\partial \Omega$ with radius $\mu$ ? Indeed, this is the case and can be derived from a theorem of J. Rauch [10, p. 502]. Before stating his result, we first give a definition.

Definition. Let $M$ and $M^{\prime}$ be two smooth compact hypersurfaces in $\mathbf{R}^{n+1}$ with positive-definite second fundamental forms II and II $^{\prime}$, respectively. We say that $M$ and $M^{\prime}$ are internally tangent at a point $p \in M \cap M^{\prime}$ if they are tangent at $p$ and have the same outward normal $v$ at $p$.

(1.1) Theorem (J. Rauch). Let $M$ and $M^{\prime}$ be as in the preceding definition, and assume that $\mathrm{II}_{p} \geq \mathrm{II}_{p}^{\prime}$, for all $p \in M, p^{\prime} \in M^{\prime}$ with $v(p)=v\left(p^{\prime}\right)$. If, in addition, $M$ and $M^{\prime}$ are internally tangent at one point, then $M$ lies inside $M^{\prime}$ in the sense that $M$ is contained in the closed bounded domain determined by $M^{\prime}$.

Let $0 \leq \mu<\frac{1}{K}$. We Define $T(\mu)$ to be the inner tubular neighborhood of $\partial \Omega$ with radius $\mu$, i.e. $T(\mu)=\{x \in \bar{\Omega} \mid \operatorname{dist}(x, \partial \Omega) \leq \mu\}$. We also define $\widetilde{T}(\mu)=\{(p,-t v(p)) \mid p \in \partial \Omega, t \in[0, \mu]\}$; then $\widetilde{T}(\mu)$ is a subset of the normal bundle of $M$. With the help of (1.1), we can prove the following result.

(1.2) Theorem. The restriction of the exponential map of the normal bundle over $M=\partial \Omega$ to $\widetilde{T}(\mu)$ is a diffeomorphism onto $T(\mu)$. More precisely, the map

defined by

$$
\exp : \widetilde{T}(\mu) \rightarrow T(\mu)
$$

is a diffeomorphism.

$$
\exp (p,-t v(p)) \equiv \exp _{p}(-t v(p))=p-t v(p)
$$

To prove (1.2), we first prove some lemmas.

(1.3) Lemma. $S(p, q, \mu) \subset \bar{\Omega}$ for all $p \in M$ and $0 \leq \mu<\frac{1}{K}$, where $S(p, q, \mu)$ is the $n$-sphere of radius $\mu$ and center at $q$ which is a point along the inner normal direction at $p$ such that $\operatorname{dist}(p, q)=\mu$.

Proof. Let $M^{\prime}=S(p, q, \mu)$. Then it is clear that $M$ and $M^{\prime}$ are internally tangent at $p$. Moreover, the second fundamental form of $M^{\prime}$ is $\frac{1}{\mu}$ times the inner product on $T_{p} M$ which is larger than that of $M$ by the choice of $\mu$. Hence $M^{\prime} \subset \bar{\Omega}$ by (1.1). This is true for all $p \in M$ and $0 \leq \mu<\frac{1}{K}$. Q.E.D.

Intuitively, the lemma says that we can roll a solid ball of any radius $<\frac{1}{K}$ along the inner boundary of $M$.

(1.4) Lemma. $S(p, q, \mu) \cap M=\{p\}$ for all $p \in M$ and $0 \leq \mu<\frac{1}{K}$.

Proof. From (1.3), we know that $S(p, q, \mu) \subset \bar{\Omega}$. If there is another point $p^{\prime} \in S(p, q, \mu) \cap M$, then $\operatorname{dist}(p, q)=\operatorname{dist}\left(p^{\prime}, q\right)=\mu$. Choose any point $q^{\prime}$ 


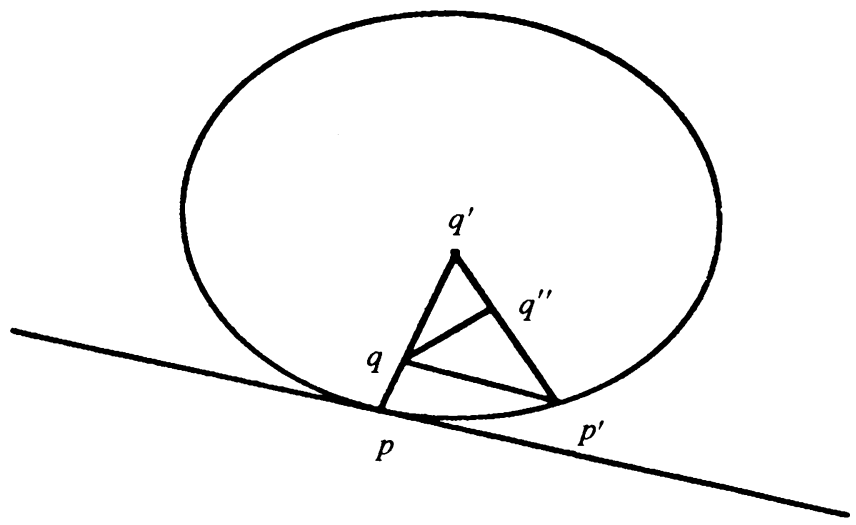

FIGURE 1

along the ray $\overrightarrow{p q}$ such that $\operatorname{dist}\left(p, q^{\prime}\right)=\mu^{\prime}$ and $\mu<\mu^{\prime}<\frac{1}{K}$. Again, by (1.1), we have $S\left(p, q^{\prime}, \mu^{\prime}\right) \subset \bar{\Omega}$. Let $q^{\prime \prime}$ be the point such that $\overline{q q^{\prime \prime}} \perp \overline{p^{\prime} q^{\prime}}$ as Figure 1 shows.

Then

$$
\begin{aligned}
\operatorname{dist}\left(p, q^{\prime}\right) & =\operatorname{dist}(p, q)+\operatorname{dist}\left(q, q^{\prime}\right)=\operatorname{dist}\left(p^{\prime}, q\right)+\operatorname{dist}\left(q, q^{\prime}\right) \\
& >\operatorname{dist}\left(p^{\prime}, p^{\prime \prime}\right)+\operatorname{dist}\left(q^{\prime \prime}, q^{\prime}\right)=\operatorname{dist}\left(p^{\prime}, q^{\prime}\right) .
\end{aligned}
$$

This implies that $\operatorname{dist}\left(p^{\prime}, q^{\prime}\right)<\mu^{\prime}$. Therefore, $S\left(p, q^{\prime}, \mu^{\prime}\right)$ cannot stay inside $\bar{\Omega}$ which contradicts (1.3). Q.E.D.

(1.5) Corollary. No two normals through $M$ intersect in $T(\mu)$; i.e. exp is injective on $\widetilde{T}(\mu)$.

Proof. Suppose that $v_{p}$ and $v_{p^{\prime}}$ are two normals through $p$ and $p^{\prime}$, respectively. If $v_{p} \cap v_{p^{\prime}}=\{q\}$ with $\operatorname{dist}(p, q)=\mu^{\prime}, \operatorname{dist}\left(p^{\prime}, q\right)=\mu^{\prime \prime}$ and $\mu^{\prime}, \mu^{\prime \prime} \leq \mu$, then we have three cases:

(i) $\mu^{\prime}>\mu^{\prime \prime}$. This implies that $S\left(p, q, \mu^{\prime}\right) \not \subset \bar{\Omega}$

(ii) $\mu^{\prime}<\mu^{\prime \prime}$. This implies that $S\left(p^{\prime}, q, \mu^{\prime \prime}\right) \not \subset \bar{\Omega}$.

(iii) $\mu^{\prime}=\mu^{\prime \prime}$. This implies that $S\left(p, q, \mu^{\prime}\right) \cap M$ contains at least two points.

(i) and (ii) contradict (1.3) and (iii) is also impossible by (1.4). Therefore, (1.5) is proved. Q.E.D.

To complete the proof of (1.2), we need the comparison theorem of E. Rauch $\left[13\right.$, p. 350]. For hypersurfaces in $\mathbf{R}^{n+1}$, we can simply formulate it as follows.

Let $M$ be a smooth compact hypersurface in $\mathbf{R}^{n+1}, p \in M, \gamma(t)$ a geodesic in $\mathbf{R}^{n+1}$ parametrized by arc length with domain the interval $[0, b]$, initial point $p$ and initial vector $\dot{\gamma}(0)=-v(p)$, where $v$ is the unit outward normal. Let $\mathrm{II}_{\dot{\gamma}(0)}$ be the second fundamental form of $M$ in the direction $\dot{\gamma}(0)$. Note that this is different from our previous conventions by a sign. Assume that we have another such setup $M^{\prime}, p^{\prime}, \gamma^{\prime}, v^{\prime}, \mathrm{II}_{\dot{\gamma}^{\prime}(0)}$ with domain of $\gamma^{\prime}$ the interval $[0, b]$. Under these assumptions, we can state the comparison theorem of Rauch as follows.

(1.6) Theorem (E. Rauch). Assume that the minimum eigenvalue of $\mathrm{II}_{\dot{\gamma}^{\prime}(0)}$ is larger than the maximum eigenvalue of $\mathrm{II}_{\dot{\gamma}(0)}$. If there are no focal points on $\gamma^{\prime}$, then there are no focal points on $\gamma$. 
In our application, $M$ will be $\partial \Omega$ and $M^{\prime}$ is the $n$-sphere with radius $\frac{1}{K}$, $b=\mu$. We adopt the previous notations. Then there are no focal points along $\gamma^{\prime}$ since $\mu<\frac{1}{K}$. Moreover, the minimum eigenvalue of $\mathrm{II}_{\hat{\gamma}_{(0)}}$ is $-K$ by the definition of $K$ and the eigenvalue of $\mathrm{II}_{\dot{\gamma}^{\prime}(0)}$ is $-\frac{1}{\mu}$. Obviously, $-K>-\frac{1}{\mu}$ by the choice of $\mu$. Therefore (1.6) implies that there are no focal points along $\gamma$. Since $\gamma$ can be arbitrary, we conclude that $d$ exp is never singular on $\widetilde{T}(\mu)$ which follows from the definition of focal point [1, p. 224]. We have therefore proved:

(1.7) Theorem. $d$ exp is never singular in $\widetilde{T}(\mu)$.

Finally, the proof of (1.2) is an obvious combination of (1.5) and (1.7).

The following is an immediate consequence of (1.7).

(1.8) Corollary. Let $\gamma(s)$ be a geodesic through $\gamma(0)=p \in M$ with $\dot{\gamma}(0) \in$ $\left(T_{p} M\right)^{\perp}$. Then the focal points of $M$ occur after $\gamma(\mu)$.

Now, we are in position to exhibit a defining function of $\Omega$. First, we define a continuous function on $\mathbf{R}^{n+1}$ as follows.

$$
\delta(x)= \begin{cases}-\operatorname{dist}(x, M) & \text { if } x \in \Omega, \\ \operatorname{dist}(x, M) & \text { if } x \notin \Omega .\end{cases}
$$

Definition. $\delta$ is called the signed distance function of $\Omega$.

(1.9) Proposition. $\delta$ is smooth in $T(\mu)$.

Proof. Define a function $\theta$ on $\widetilde{T}(\mu)$ by $\theta(x, t v(x))=t$. Then $\theta$ is obviously smooth. Since $\exp ^{-1}$ is well defined on $T(\mu)$ by (1.2) and it is clear that

$$
\delta=\theta \circ \exp ^{-1} \text {, }
$$

$\delta$ is smooth on $T(\mu)$. Q.E.D.

(1.10) Proposition. $\|\operatorname{grad} \delta(x)\|=1$ for all $x \in T(\mu)$.

Proof. This follows from the exponential map preserving distance in the radial direction and $\|\operatorname{grad} \theta\|=1$. Q.E.D.

So far, we have obtained a defining function $\delta$ for $\Omega$ by the very definition of $\delta$ and (1.10) except that $\delta$ is not smooth on the whole $\mathbf{R}^{n+1}$. But, for our later applications, we can extend $\delta$ smoothly to $\mathbf{R}^{n+1}$ in an arbitrary way as long as it is a defining function and coincides with $\delta$ in a small neighborhood of $T(\mu)$. We still use $\delta$ to denote such an extension.

In order that $\delta$ be useful, we have to compute the Hessian of $\delta$.

Definition. The Hessian of a real-valued, smooth function $f$ on $\mathbf{R}^{n+1}$ is, by definition,

for all $X, Y \in \mathbf{R}^{n+1}$.

$$
D^{2} f(X, Y)=X(Y f)-\left(D_{X} Y\right) f
$$

It is well known that $D^{2} f$ is a symmetric bilinear form on $\mathbf{R}^{n+1}$. Moreover, if $e_{1}, \ldots, e_{n+1}$ is an orthonormal basis of $\mathbf{R}^{n+1}$, then the eigenvalues of the matrix

$$
H_{f}(x)=\left[D^{2} f\left(e_{i}, e_{j}\right)\right]
$$

are independent of the choice of $e_{1}, \ldots, e_{n+1}$ at $x$.

Now, we can compute the eigenvalues of $H_{\delta}$ in terms of the principal curvatures of $M=\partial \Omega$. Recall that $\mu<\frac{1}{K}$. We have 
(1.11) Theorem. Let $x_{0} \in T(\mu)$ and $y_{0}$ be the unique point in $M$ such that $\delta\left(x_{0}\right)=-\operatorname{dist}\left(x_{0}, y_{0}\right)$. Then, relative to a suitable orthonormal basis in the tangent space to $y_{0}$, we have

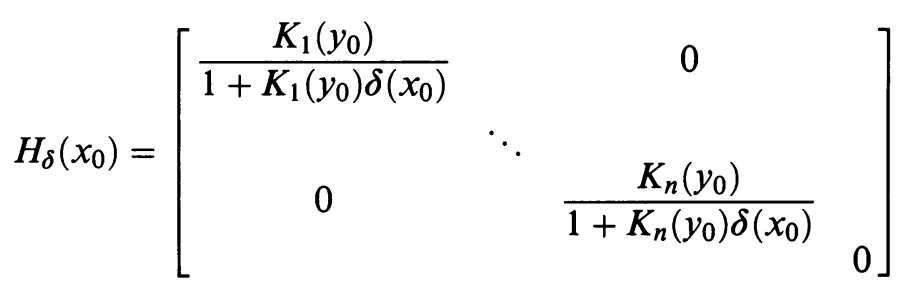

where $K_{1}, \ldots, K_{n}$ are the principal curvatures of $M$ at $y_{0}$.

Proof. The proof can be found in [3, p. 383]. The only difference is that we have defined $\mu$ in terms of the principal curvatures of $M$. Q.E.D.

Remark. If $x_{0} \in M$, then

$$
H_{\delta}\left(x_{0}\right)=\left[\begin{array}{cccc}
K_{1}\left(x_{0}\right) & & 0 & \\
& \ddots & & \\
0 & & K_{n}\left(x_{0}\right) & \\
& & & 0
\end{array}\right]
$$

is nothing but the second fundamental form of $M$ at $x_{0}$ with respect to the principal directions. In general, $H_{\delta}\left(x_{0}\right)$ is the second fundamental form at $x_{0}$ of the hypersurface defined by $\delta(x)+\varepsilon=0$, where $0 \leq \varepsilon \leq \mu$ and $\varepsilon=$ $\operatorname{dist}\left(x_{0}, M\right)$.

In view of (1.11), the Hessian of $\delta$ degenerates in one direction. However, we can easily modify $\delta$ so that the resulting function has positive definite Hessian everywhere on $T(\mu)$. Define

$$
\rho(x)=\left[\frac{1}{K}+\delta(x)\right]^{2}-\frac{1}{K^{2}} .
$$

Then we have the following basic properties about $\rho$.

(1.12) Proposition.

(1) $\rho$ is a smooth defining function for $\Omega$.

(2) $d \rho(x)=2\left[\frac{1}{K}+\delta(x)\right] d \delta(x)$.

(3) $2\left[\frac{1}{K}-\mu\right] \leq\left\|d_{\rho}(x)\right\| \leq 2 \cdot \frac{1}{K}$ for all $x \in T(\mu)$.

(4) $\rho$ is a strictly convex function near $T(\mu)$; i.e., $H_{\rho}(x)$ is positive definite everywhere on $T(\mu)$. In fact,

$$
H_{\rho}(x)=2\left[\left(\frac{1}{K}+\delta(x)\right) H_{\delta}(x)+d \delta(x) \otimes d \delta(x)\right] .
$$

(5) For $x \in T(\mu)$ and $\xi \in \mathbf{R}^{n+1}$, we have

$$
k\left[\frac{1}{K}-\mu\right] \leq \frac{1}{2} H_{\rho}(x)(\xi, \xi) \leq\|\xi\|^{2} .
$$

Proof. It suffices to prove (4) and (5) since the remaining parts are obvious. 
From (1.11), for $x \in T(\mu)$, we have

$$
H_{\delta}(x)=\left[\begin{array}{ccc}
\frac{K_{1}(y)}{1+K_{1}(y) \delta(x)} & & 0 \\
0 & \ddots & \frac{K_{n}(y)}{1+K_{n}(y) \delta(x)} \\
& & 0
\end{array}\right]
$$

w.r.t. a suitable orthonormal basis $\left\{e_{1}, \ldots, e_{n}\right\}$ of $T_{y} M$, where $y$ is the unique point in $M$ such that $\delta(x)=-\operatorname{dist}(x, y)$. An elementary computation gives

$$
H_{\rho}(x)=2\left[\left(\frac{1}{K}+\delta(x)\right) H_{\delta}(x)+d \delta(x) \otimes d \delta(x)\right] .
$$

So, w.r.t. the basis $\left\{e_{1}, \ldots, e_{n}, v\right\}$, we have

$$
H_{\delta}(x)=\left[\begin{array}{ccc}
\frac{\left(\frac{1}{K}+\delta(x)\right) K_{1}(y)}{1+K_{1}(y) \delta(x)} & 0 \\
0 & \ddots & \frac{\left(\frac{1}{K}+\delta(x)\right) K_{n}(y)}{1+K_{n}(y) \delta(x)} \\
& & 1
\end{array}\right]
$$

This proves (4).

For each $i$, we have

$$
\frac{\left(\frac{1}{K}+\delta(x)\right) K_{i}(y)}{1+K_{i}(y) \delta(x)} \leq \frac{1+K_{i}(y) \delta(x)}{1+K_{i}(y) \delta(x)}=1,
$$

where we use $K_{i} \leq K$ in the inequality. So all the eigenvalues of $H_{\rho}(x)$ are bounded by 1 . For the lower bound, we observe that, for all $i$,

$$
1+K_{i}(y) \delta(x) \leq 1 \text { on } T(\mu) .
$$

Therefore,

$$
\frac{\left(\frac{1}{K}+\delta(x)\right) K_{i}(y)}{1+K_{i}(y) \delta(x)} \geq\left(\frac{1}{K}+\delta(x)\right) K_{i}(y) \geq k\left(\frac{1}{K}-\mu\right) .
$$

This is true for all $x \in T(\mu)$ and $i$. Moreover, $k\left(\frac{1}{K}-\mu\right) \leq \frac{k}{K} \leq 1$. Hence, the lower bound for the eigenvalues is $k\left(\frac{1}{K}-\mu\right)$. This implies (5). Q.E.D.

Remark. So far, we always assume $\mu<\frac{1}{K}$. For later purposes, we will take $\mu$ to be of the form $\frac{s}{K}$, where $0<s<1$ and is fixed. Define $m=\frac{k}{K}$. Then (3) and (5) of (1.12) give the following.

(1.13) Corollary. For $x \in T(\mu)$ and $\xi \in \mathbf{R}^{n+1}$, we have

(1) $2(1-s) \cdot \frac{1}{K} \leq\|d \rho(x)\| \leq 2 \cdot \frac{1}{K}$.

(2) $(1-s) m\|\xi\|^{2} \leq \frac{1}{2} H_{\rho}(x)(\xi, \xi) \leq\|\xi\|^{2}$.

We have completed the construction of a defining function for $\Omega$ and have estimated its gradient and Hessian in terms of the maximum and minimum eigenvalues of the second fundamental form of $\partial \Omega$. 
2. AN APPLICATION TO THE UNIFORM ESTIMATE OF THE $\bar{\partial}$-PROBLEM

Let $\Omega$ be a smooth, bounded strictly convex domain in $\mathbf{C}^{n}, n \geq 2$, and

$$
f(z)=\sum_{j=1}^{n} f_{j}(z) d \bar{z}_{j} \in C_{(0,1)}^{\infty}(\bar{\Omega}),
$$

i.e., a $\bar{\partial}$-closed $(0,1)$-form which is defined and smooth near $\bar{\Omega}$. From $[2,5]$, Henkin's solution of $\bar{\partial} u=f$ on $\Omega$ is given by

$$
\begin{aligned}
\left(H_{\Omega} f\right)(z)= & \frac{1}{n W(n)} \int_{\partial \Omega \times[0,1]} f(\xi) \wedge \omega^{\prime}(\eta) \wedge \omega(\zeta) \\
& -\frac{1}{n W(n)} \int_{\Omega} f(\zeta) \wedge \omega^{\prime}(u) \wedge \omega(\zeta)
\end{aligned}
$$

for $z \in \Omega$, where

$$
\begin{aligned}
\omega(\zeta) & =d \zeta_{1} \wedge \cdots \wedge d \zeta_{n}, \\
\eta & =\left(\eta_{1}, \ldots, \eta_{n}\right), \quad u=\left(u_{1}, \ldots, u_{n}\right), \quad v=\left(v_{1}, \ldots, v_{n}\right), \\
\eta_{j} & =\lambda u_{j}+(1-\lambda) v_{j}, \quad \lambda \in[0,1], \quad j=1, \ldots, n, \\
\omega^{\prime}(\eta) & =\sum_{j=1}^{n}(-1)^{j+1} \eta_{j} d \eta_{1} \wedge \cdots \wedge d \hat{\eta}_{j} \wedge \ldots \wedge d \eta_{n}, \\
u_{j} & =\frac{\bar{\zeta}_{j}-\bar{z}_{j}}{|\zeta-z|^{2}}, \quad j=1, \ldots, n, \\
v_{j}= & \frac{-1}{\Phi} \frac{\partial \rho(\zeta)}{\partial z_{j}}, \quad j=1, \ldots, n, \\
\Phi(z, \zeta) & =\sum_{j=1}^{n} \frac{\partial \rho(\zeta)}{\partial z_{j}}\left(z_{j}-\zeta_{j}\right)
\end{aligned}
$$

and satisfies

$$
\bar{\partial}\left(H_{\Omega} f\right)(z)=f(z) \text { on } \Omega, \quad\left\|H_{\Omega} f\right\|_{L^{\infty}(\Omega)} \leq C_{\Omega}\|f\|_{L^{\infty}(\Omega)} .
$$

The constant $C_{\Omega}$ depends, of course, on the domain only. However, the explicit estimate of $C_{\Omega}$ in general seems impossible as we mention in $\S 0$. For a strictly convex domain, (2.1) involves the first and second order derivatives of $\rho$ and we already have the estimates of $d \rho(\zeta)$ and $H_{\rho}(\zeta)$ obtained in $\S 1$. So we will be able to compute $C_{\Omega}$ explicitly in terms of the geometric invariants of $\Omega$. In fact, we have

(2.2) Theorem. Let $\Omega$ be a bounded smooth strictly convex domain in $\mathbf{C}^{n}$, $n \geq 2, f \in C_{(0,1)}^{\infty}(\bar{\Omega})$ and $\bar{\partial} f=0$ on $\Omega$. Then Henkin's solution defined in (2.1) satisfies

$$
\left\|H_{\Omega} f\right\|_{L^{\infty}(\Omega)} \leq \frac{2}{C_{1} \omega_{2 n-1}}\left[C_{1} R \omega_{2 n-1}+C_{2} \operatorname{vol}(\partial \Omega)+C_{3} \omega_{2 n-3}\right]\|f\|_{L^{\infty}(\Omega)},
$$

where $C_{1}=C_{1}(n, K), C_{2}=C_{2}(n, s, R, k, K), C_{3}=C_{3}(n, s, k, K), 0<$ $s<1, R=$ diameter of $\Omega, \omega_{2 n-1}=\operatorname{vol}\left(S^{2 n-1}\right), \omega_{2 n-3}=\operatorname{vol}\left(S^{2 n-3}\right), k$ and $K$ are the minimum and maximum principal curvatures of $\partial \Omega$, respectively.

The complete proof of (2.2) and the explicit expressions of $C_{1}, C_{2}$ and $C_{3}$ can be found in [2]. Here, we only sketch its main idea: To get the explicit 
estimate, the main step is to estimate the Henkin kernel. With the help of the explicit estimates of $d \rho(x)$ and $H_{\rho}(x)$ (1.13) and a series of calculations [2], we end up with the following inequality:

$$
\left\|H_{\Omega} f\right\|_{L^{\infty}(\Omega)} \leq(2 R+C(n, K) \cdot I)\|f\|_{L^{\infty}(\Omega)},
$$

where $R=\operatorname{dia}(\Omega), K$ is the maximum principal curvature of $\partial \Omega, C(n, K)$ is a constant depending on $n, K$ and

$$
I=\int_{\partial \Omega} \frac{\left(1+2 \frac{|\zeta-z|^{2}}{|\Phi|}\right)^{n-2}}{|\Phi||\zeta-z|^{2 n-3}} d \sigma(\zeta)
$$

The next step is to analyze the integrand in $I$ and get upper bounds for $I$ according to the position of $z$ in $\Omega$. The difficult part is when $z$ approaches $\partial \Omega$. In this case, we are forced to consider a small ball $B(z ; r)$ around $z$ where $r=s^{2} / 16 n K$ and $0<s<1$. Again following Henkin's ideas and (1.13), we can define explicitly $2 n$ real-valued functions $t_{1}, \ldots, t_{2 n}$ on $B(z ; r)$ and show that, with the choice of $r,\left(t_{1}, \ldots, t_{2 n}\right)$ defines a new coordinate system on $B(z ; r)$. So, we can work on this new coordinate system and get an upper bound for $I$, thereby completing the proof of (2.2).

By the Ascoli-Arzela theorem and the diagonalization process, we can remove the condition of $f$ being continuous on $\bar{\Omega}$ and obtain the following result.

(2.3) Theorem. Let $\Omega$ be a bounded smooth strictly convex domain in $\mathbf{C}^{n}$, $n \geq 2, f \in C_{(0,1)}^{\infty}(\Omega)$ and $\bar{\partial} f=0$ on $\Omega$. Then there exists a function $u \in$ $C^{\infty}(\Omega)$ such that

$$
\bar{\partial} u=f \text { on } \Omega \text { and }\|u\|_{L^{\infty}(\Omega)} \leq C_{\Omega}\|f\|_{L^{\infty}(\Omega)},
$$

where $C_{\Omega}$ is the constant defined in (2.2).

Finally, a remark we should make is that, in the case of strongly pseudoconvex domains, the proof of (2.3) must employ a stability result which is not so obvious to obtain [9, p. 409 and the references there]. Roughly speaking, it says that the constant $C_{\Omega}$ cannot change too much under smooth perturbation of the domain, and hence the limit process can go through. In our case, not only do we get an explicit constant, but we also prove the stability result in the case of strictly convex domains.

\section{ACKNOWLEDGMENT}

The author thanks the referees for their useful comments in revising the paper.

\section{BIBLIOGRAPHY}

1. R. L. Bishop and R. J. Crittenden, Geometry of manifolds, Academic Press, New York, 1964.

2. T. G. Chen, On Henkin's solution of the $\bar{\partial}$-problem on strictly convex domains in $\mathbf{C}^{n}$, Ph.D. Thesis, Univ. of California at Berkeley, 1985.

3. S. Gilbarg and N. Trudinger, Elliptic partial equations of second order, Springer, Berlin, 1977.

4. H. Grauert and I. Lieb, Das Ramirezsche Integral und die Lösung der Gleichung $\bar{\partial} f=\alpha$ im Bereich der beschränkten Formen, Rice Univ. Studies 56 (1970), 29-50. 
5. G. M. Henkin, Integral representations of functions holomorphic in strictly pseudoconvex domains and applications to the $\bar{\partial}$-problem, Mat. Sb. 82 (124) (1979), 300-308; English transl., Math. U.S.S.R.-Sb. 11 (1970), 273-281.

6. L. Hörmander, $L^{2}$ estimates and existence theorems for the $\bar{\partial}$ operator, Acta Math. 113 (1965), 82-152.

7. __ Introduction to complex analysis in several variables, North-Holland, Amsterdam, 1973.

8. N. Kerzman, Hölder and $L^{p}$ estimates for solution of $\bar{\partial} u=f$ on strongly pseudoconvex domains, Comm. Pure. Appl. Math. 24 (1971), 301-380.

9. S. G. Krantz, Function theory of several complex variables, 2nd ed., Wadsworth \& Brooks, Pacific Grove, CA, 1992.

10. J. Rauch, An inclusion theorem for ovaloids with comparable second fundamental forms, J. Differential Geom. 9 (1974), 501-505.

11. R. Sacksteder, On hypersurfaces with no negative sectional curvatures, Amer. J. Math. 82 (1960), 609-630.

12. N. Sibony, Un exemple de domaine pseudoconvexe regulier où l'équation $\bar{\partial} u=f$ n'admet pas de solution bornée pour f bornée, Invent. Math. 62 (1980), 235-242.

13. F. W. Warner, Extension of the Rauch comparison theorem to submanifolds, Trans. Amer. Math. Soc. 122 (1966), 341-356.

Department of Mathematical Sciences, National Chengchi University, Taipei, TaIWAN, ROC 